\title{
Rehabilitación oral compleja en un paciente comprometido periodontalmente. Reporte de caso clínico y seguimiento por 6 años
}

\author{
Oral complex rehabilitation in periodontally compromised patient. Clinical case report and follow-up for 6 years \\ Jeanette Gonzales-Mattos 1,a,b,c,d, Arturo Kobayashi Shinya ${ }^{2, a, b, d, e, ~}$, Martin Quintana del Solar 2, a,b,d,e
}

\begin{abstract}
RESUMEN
La rehabilitación oral de pacientes comprometidos periodontalmente nos plantea un desafío clínico ya que conlleva muchos pasos sensibles y sobre todo el compromiso del paciente para cumplir un plan de mantenimiento adecuado. Por lo tanto, para alcanzar los resultados esperados es necesario e imprescindible un tratamiento multidisciplinario que involucre especialidades que trabajen bajo un plan de tratamiento coordinado. Con la introducción de los implantes dentales se ha proporcionado beneficios a los pacientes llevando a mejorar la calidad de vida del paciente así como su grado de satisfacción.

En el presente artículo se relata el caso clínico de un paciente periodontalmente comprometido, su tratamiento y seguimiento por seis años hasta alcanzar el éxito del mismo con una rehabilitación implanto soportada tipo prótesis híbrida en el maxilar superior y coronas cementadas sobre implantes en el maxilar inferior siguiendo un protocolo que nos brinda predictibilidad y una alta satisfacción del paciente.
\end{abstract}

PALABRAS CLAVE: Prótesis e implantes, satisfacción del paciente, periodontitis.

\section{SUMMARY}

The oral rehabilitation of periodontally compromised patients present a clinical challenges because takes many sensible steps, especially the patient's commitment to perform a maintenance plan. Therefore, in order to achieve the expected results, a multidisciplinary treatment involving specialties that work under a coordinated treatment plan is essential. The introduction of dental implants has provided benefits to leading to improving the quality of life and the satisfaction of the patient. In the present article a clinical case of a periodontal compromised patient is related, treatment and follow-up for six years to the success of such treatment an implant supported rehabilitation type hybrid prosthesis in the maxilla and crowns cemented on implants in the lower jaw is reported following a protocol that provides us with adequate treatment predictability and high patient satisfaction.

KEYWORDS: Prostheses and implants, patient satisfaction, periodontitis.

EAP en Odontologia, especialidad de rehabilitación Oral, Universidad Privada Norbert Wiener. Lima, Perú.

Departamento Académico de Clínica, Facultad de Estomatología, Universidad Peruana Cayetano Heredia. Lima, Perú.

Cirujano Dentista.

Especialista en Rehabilitación Oral,

Magister en Rehabilitación Oral.

Docente

Magister en Estomatología 


\section{INTRODUCCIÓN}

La predisposición a la enfermedad periodontal es causada principalmente por el pobre control de placa bacteriana, por lo que es necesario, antes de iniciar un tratamiento rehabilitador realizar un control y seguimiento de la enfermedad (1).

El edentulismo está directamente relacionado con importantes alteraciones anatómicas, funcionales y psicológicas de los pacientes. La falta de estabilidad y de retención de las prótesis convencionales, asociada a la capacidad reducida de masticación puede reducir la calidad de vida de los pacientes (2).

Un plan de tratamiento implantosoportado debe ser realizado considerando puntos anatómicos así como la relación que tendrá con el habla, la masticación y la estética (3), la mejoría en la calidad de vida (4) y el grado de satisfacción de los pacientes (5), además para seleccionar el adecuado tipo de prótesis se deben considerar algunos factores durante el examen clínico considerados en el protocolo reverso $(6,7)$.

Una de las alternativas es el uso de prótesis hibridas para edéntulos totales (8) y prótesis unitarias sobre implantes para edéntulos parciales, atornillada o cementada (9).

El propósito del presente caso clínico es describir la rehabilitación de un paciente comprometido periodontalmente con prótesis implanto soportadas tipo prótesis híbrida superior y prótesis cementadas unitarias en el sector postero inferior, y sus controles por 6 años.

\section{CASO CLÍNICO}

Paciente masculino de 63 años de edad en aparente buen estado general, acude al Servicio del Postgrado de Rehabilitación Oral de la Facultad de Estomatología de la Universidad Peruana Cayetano Heredia (UPCH) en el año 2009 por requerir un tratamiento integral, solicitando un tratamiento fijo y estético.

Se realizarón los exámenes clínicos, radiográficos y tomográficos necesarios llegando a los siguientes diagnósticos: Periodontitis crónica localizada moderada en las piezas $12,22,35$ y severa en la pieza 15 , deformidades y condiciones mucogingivales en rebordes edéntulos: Seibert III: Sextante IV y VI. Lesiones cariosas en piezas: $13,22,24,25$, pérdida de sustancia dental causada por: Piezas talladas sin provisionales en piezas 34,35,44,15,12,21,22,24,25. Destrucción coronaria pieza 13, Diagnóstico pulpar: tratamiento de conductos previo piezas 15, 13, 12, 22, 24, Movilidad dental grado 3 en la pieza 15. Asimismo presentó un Desorden Funcional Oclusal teniendo como características: Edéntulo parcial: superior e inferior, Alteración de la guía anterior y disminución de dimensión vertical. (figura $1 \mathrm{a}$ y b).

Planteamos los objetivos del tratamiento de acuerdo a la expectativa del paciente y al pronóstico de las piezas remanentes que recibimos de las especialidades de periodoncia y endodoncia. La planificación del tratamiento se realizó en 3 fases, dando énfasis a la primera fase de estabilización y control de la enfermedad periodontal, además se trabajó en lograr una adecuada concientización del paciente.

Se optó por una rehabilitación fija e implanto soportada tanto en el maxilar superior e inferior.

La primera fase tenía como objetivo desfocalizar el área, brindarle una adecuada dimensión vertical, estabilización de la oclusión y control de la enfermedad periodontal.

Luego de 6 meses se procedió a reevaluar el caso (2da fase), realizar el protocolo reverso y observar las condiciones tomográficas de los tejidos remanentes, durante todo este tiempo el paciente fue citado en la especialidad de Periodoncia para mantener los tejidos periodontales y un IHO óptimo.

De acuerdo al protocolo reverso y al examen tomográfico (figura 2) con las guías multifuncionales mantuvimos la planificación inicial de realizar una prótesis híbrida superior implanto soportada sobre 6 implantes y 3 implantes en el maxilar inferior (figura 3 ),

Debido a que en el maxilar superior se usó hueso liofilizado y al bajo torque del implante a nivel de la pieza 23 se esperó la oseointegración por 6 meses para proceder a la rehabilitación implantosoportada (3era fase). En el maxilar superior se probaron y torquearon los mini pilares $\mathrm{CM}$, con un torque de $15 \mathrm{~N}$ para mini pilares angulados y $32 \mathrm{~N}$ para mini pilares rectos.

Se procedió a la transferencia de los pilares en el maxilar superior con cubeta abierta y ferulización de transfer (figura 4), y a la transferencia de la platafor- 
ma de los implantes en el maxilar inferior.

Luego se procedió a la fabricación de la estructura metálica del maxilar superior con la ayuda de una pantalla de silicona obtenida del protocolo reverso, esta estructura presentó un asentamiento pasivo comprobado clínica y radiográficamente luego de realizar los cortes y soldajes necesarios (figura 5). Se realizó el enfilado para la prótesis híbrida con dientes Ivostar/Gnathostar donde se evaluó estética y fonación. En el maxilar inferior se torquearon los pilares $\mathrm{CM}$ recto para la pieza 35 y Pilares angulados $\left(17^{\circ}\right)$ a nivel de piezas 36 y 46 fresados con la ayuda de una guía de colocación de pilares (figura 6) y se fabricaron coronas de metal cerámica. Para finalizar se procede a la instalación de la prótesis híbrida superior, la cementación de las coronas con cemento temporal y la instalación de una férula de DCM (figura a y b). Finalizado el tratamiento se realizaron controles periodontales y protésicos periódicos donde se comprobó la correcta concientización del paciente en cuanto a su mantenimiento. (figura 8)

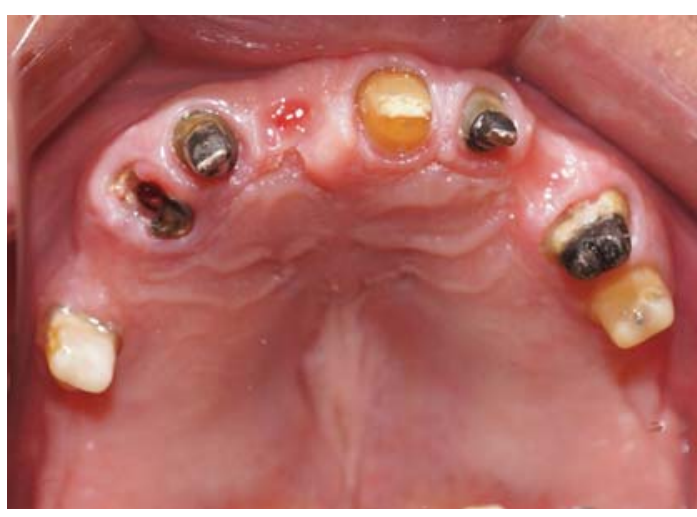

Figura 1. a: Aspecto clínico del maxilar superior.

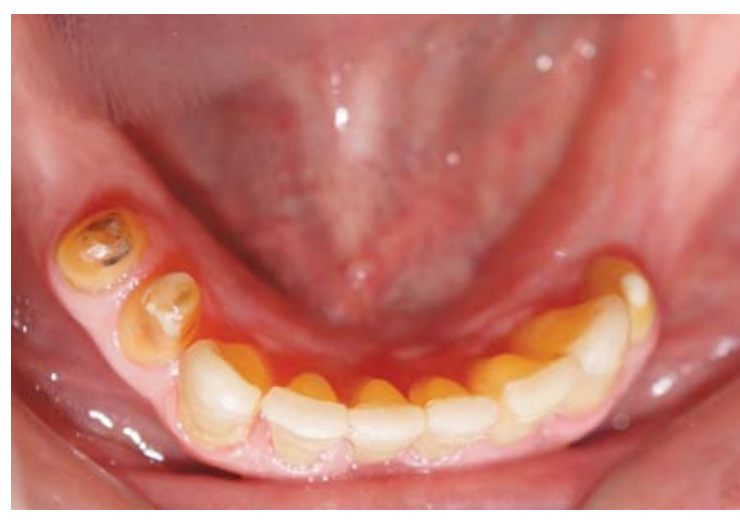

Figura 1. b: Aspecto clínico del maxilar inferior.

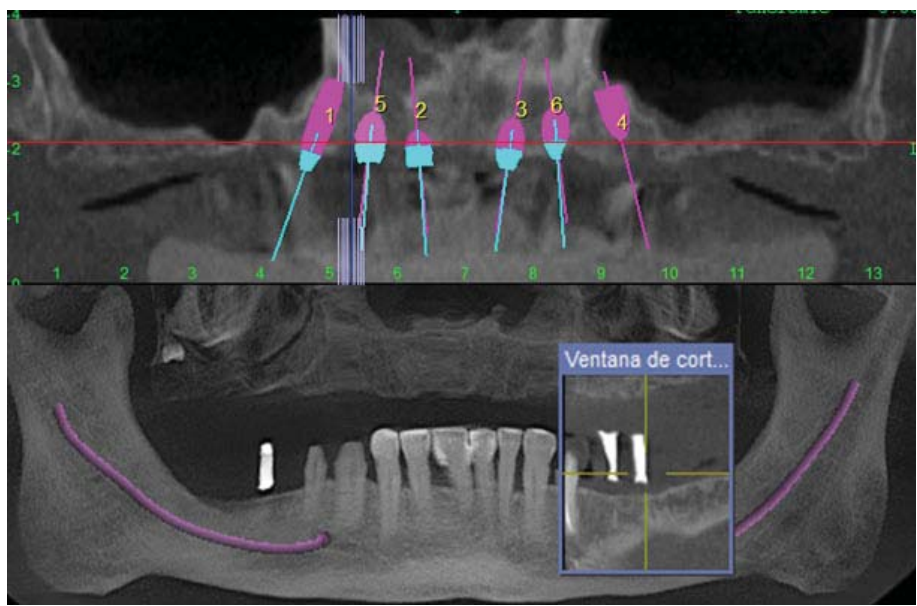

Figura 2. Tomografía del maxilar superior e inferior. 

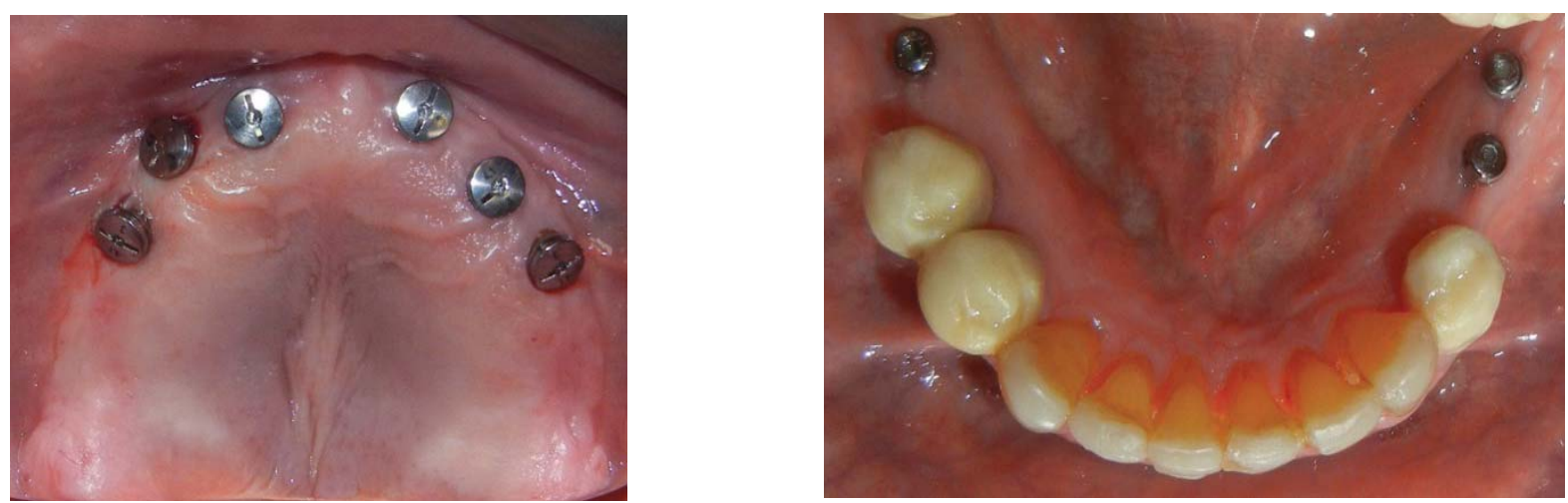

Figura 3. a y b. Colocación de tornillos de cicatrización.

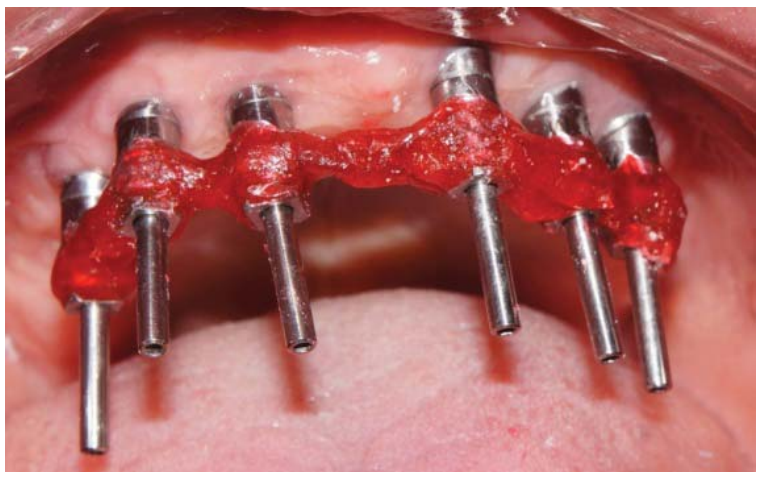

Figura 4. Ferulización de transfers

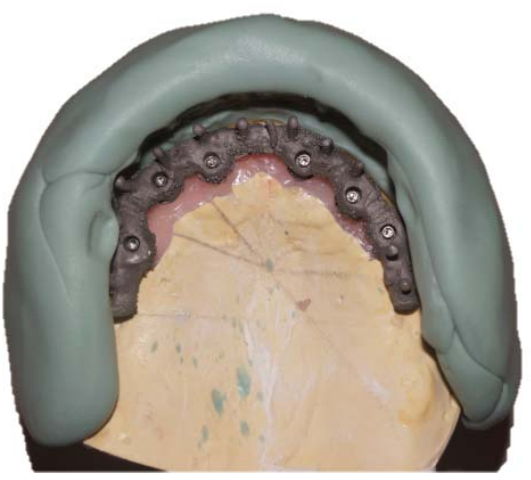

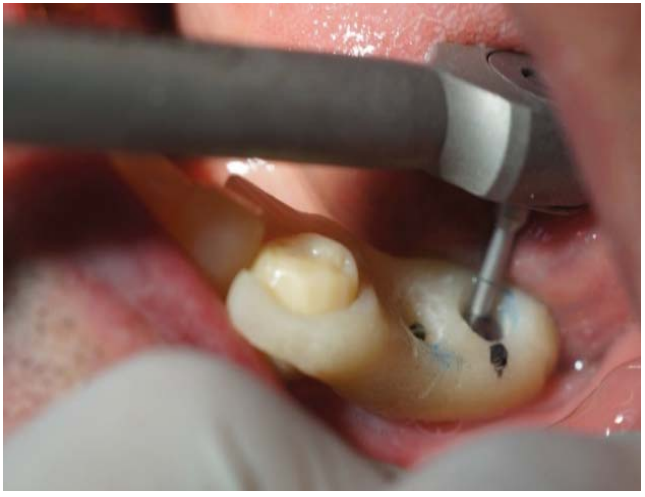

Figura 6. En el maxilar inferior se procedió al torque de los pilares con la ayuda de una guía de localización

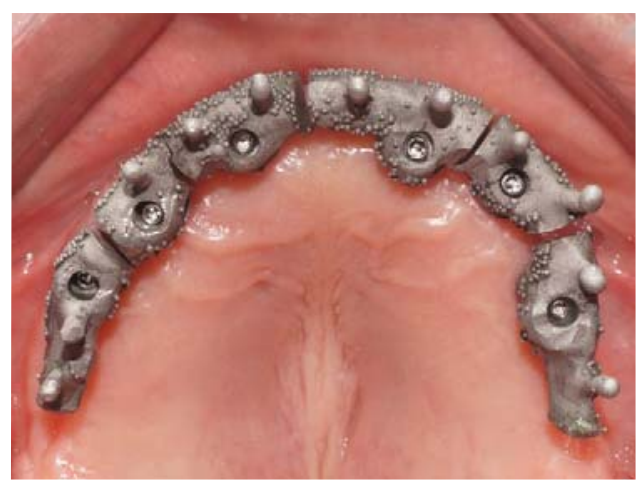

Figura 5. a y b Asentamiento pasivo de la estructura metálica sobre los pilares 


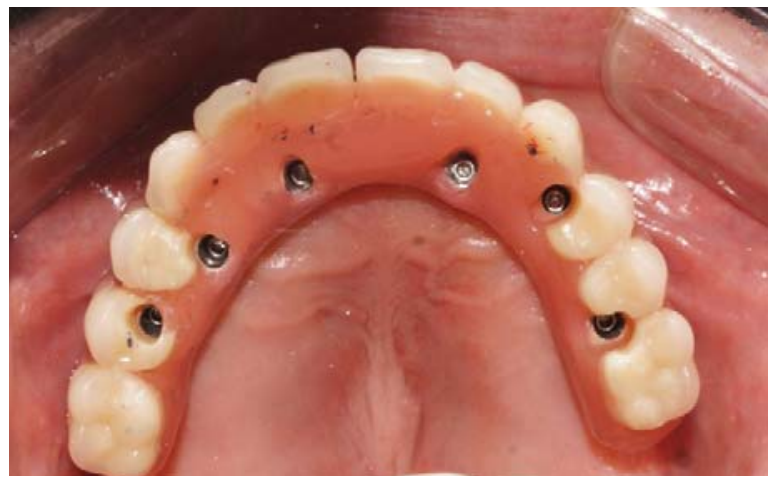

Figura 7. a Instalación de la prótesis híbrida superior

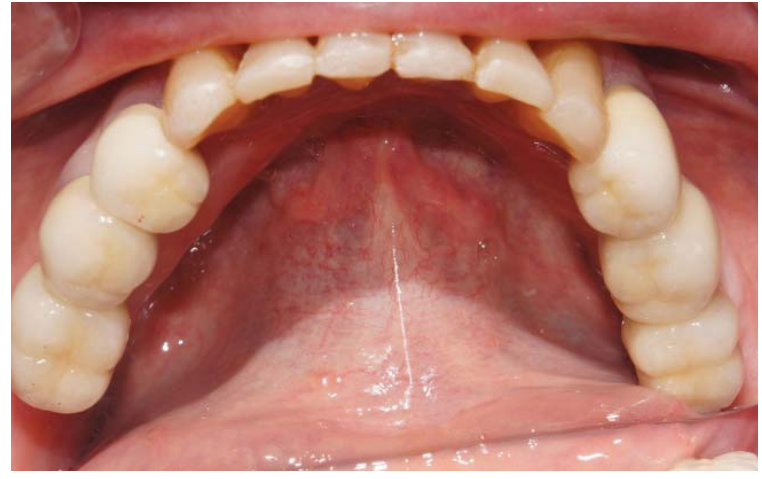

Figura 7. a Instalación de la prótesis híbrida superior

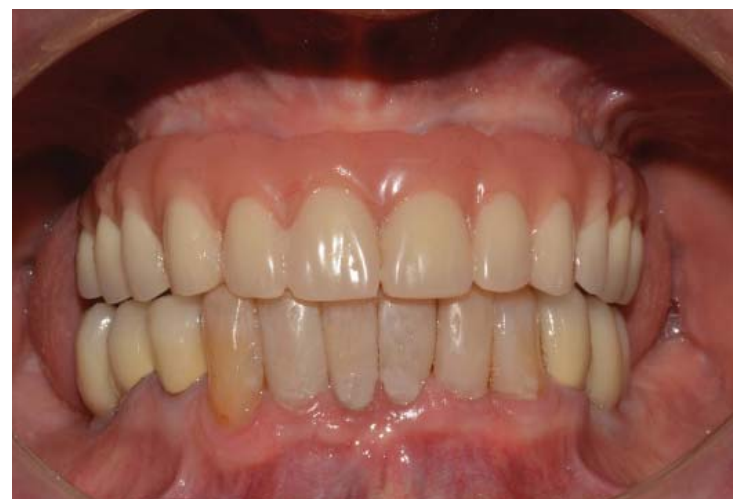

Figura 7. b coronas cementadas sobre implantes en Max. Inferior

\section{DISCUSIÓN}

La periodontitis es una enfermedad infecciosa microbiana responsable de la pérdida de dientes a nivel global. La rehabilitación oral de los pacientes comprometidos periodontalmente requiere especial atención, considerando el reducido soporte periodontal alrededor de las piezas pilares y la posible transmisión de patógenos periodontales de los dientes a los implantes, lo cual puede afectar la supervivencia a largo plazo y el éxito del tratamiento protético realizado (1). Además una baja concientización sobre la enfermedad contribuirá a .la falla de la terapia periodontal y por ende el protésico (10).

El tratamiento con implantes en pacientes periodontalmente comprometidos es viable y demuestra altos índices de éxitos siempre y cuando se cumplan requisitos como un adecuado diseño de la prótesis, adaptación marginal de esta y control de la higiene del paciente como prioridad del tratamiento (11).
El uso de implantes dentales como forma de retención para prótesis representa una opción de tratamiento previsible para la rehabilitación de pacientes edéntulos totales y parciales (12). De acuerdo al protocolo reverso y a la toma tomográfica decidimos una prótesis hibrida superior sobre 6 implantes, siendo los distales inclinados para disminuir la distancia AP y disminuir la sobrecarga (13). Esta prótesis híbrida consta de una estructura metálica que se asienta pasivamente sobre los mini pilares $(14,15)$, y una estructura de acrílico con dientes sobre ella, con una alta tasa de supervivencia $(93,3-100 \%)(16)$.

Es reconocido que las prótesis parciales removibles son la mejor opción terapéutica no quirúrgica para los espacios edéntulos, sin embargo también se sabe que ocasionalmente puede contribuir a acortar la longevidad de los pilares debido a la sobrecarga oclusal como lo mencionó Yamazaki et al., en un estudio retrospectivo comparativo de diez años, concluyó 
que la rehabilitación con prótesis fijas sobre implantes puede reducir la incidencia de pérdida de piezas en casos de edentulismo parcial (17), por ese motivo consideramos la colocación de implantes unitarios y coronas cementadas en el maxilar inferior con la colocación del pilar yuxtagingival para evitar complicaciones con el cemento (18), considerando el estudio de Linkevicius et al., quienes realizaron un análisis retrospectivo de casos para analizar si el cemento residual en coronas cementadas sobre implantes causa enfermedad periodontal concluyó que los tejidos bajo las coronas cementadas sobre implantes son mas propensas a desarrollar periodontitis si existiese cemento remanente (19).

Finalmente el esquema oclusal que se le dio para el caso fue una Oclusión mutuamente protegida, dejando libre de contactos en las desoclusiones a las coronas sobre implantes inferiores (20).

\section{CONCLUSIONES}

Los tópicos tratados en este caso clínico constituyen una guía de las consideraciones clínicas para el manejo odontológico de pacientes tratados periodontalmente y la importancia de lograr una adecuada concientización del paciente antes de iniciar el tratamiento.

El uso de implantes oseointegrados pueden ser utilizados con seguridad en pacientes con historia de enfermedad periodontal siempre y cuando esta sea controlada, y además tengamos el compromiso del paciente en realizar todos los controles periodontales que se soliciten.

Es importante considerar que mediante el uso de técnicas predecibles como el protocolo reverso y una adecuada planificación quirúrgica y protésica lograremos la completa satisfacción del paciente tanto en lo funcional como en la estética y fonética.

\section{Agradecimientos:}

A la Dra Maribel Salinas C. Área de Rehabilitación Oral-UPCH y a la Dra. Karen Yaya P. Área de Periodoncia-UPCH.

\section{Declaración de financiamiento y de conflictos de interés:}

Los autores declaran mediante juramento, que el estudio fue financiado por los autores, y declaran no tener conflictos de intereses con persona o institución alguna, ni de orden económico, ni institucional, ni laboral, ni personal

\section{Correspondencia:}

Jeanette Gonzales Mattos

Correo electrónico: jym42@hotmail.com

\section{REFERENCIAS BIBLIOGRAFICAS}

1. Dhingra K. Oral rehabilitation considerations for partially edentulous periodontal patients. Journal Of Prosthodontics. 2012; 21(6): 494-513.

2. De Kok IJ, Chang KH, Lu TS, Cooper LF.Comparison of three-implant-supported fixed dentures and twoimplant-retained overdentures in the edentulous mandible: a pilot study of treatment efficacy and patient satisfaction. Int J Oral Maxillofac Implants. 2011;26(2):415-263.

3. Bergamo E, Barbi F, Farahr G, Celestrino M, Pereira J, De Melo M. Reabilitação de maxila edêntula com prótese fixa implantossuportada: relato de caso. Dental Press Implantology. 2014; 8(4): 16-26.

4. Pjetursson BE, Karoussis I, Bürgin W, Brägger U. Lang NP. Patients satisfaction following implant therapy. A 10-year prospective cohort study. Clin Oral Implants Res. 2005;16(2):185-93.

5. Prado A, Teixeira K, Filho G, Volpato C, De Vasconcellos D. Avaliação da experiência e do grau de satisfação de pacientes tratados com próteses totais fixas sobre implantes. Dental Press Implantology. 2014. 8(4): 60-67.

6. Almeida H, De Santana E, Dos Santos N, De Moraes P, De Araújo Y, de Martinez G M. Clinical aspects in the treatment planning for rehabilitation with overdenture and protocol-type prosthesis. RGO. 2015; 63(3): 271276.

7. Mattos da Silveira B, Guedes M, Claudino M, Bernardes S. Planejamento reverso para tratamento com carga imediata. J ILAPEO. 2011; 5 (3) : 79-83.

8. Drago C, Gurney L. Maintenance of Implant Hybrid Prostheses: Clinical and Laboratory Procedures. J Prosthodont .2013; 22(1): 28-35.

9. Shadid R, Sadaqa N. A Comparison between screwand cement-retained implant prostheses: A literature review. J Oral Implantol. 2012; 38(3): 298-307.

10. George A, George T, Vishnupriya V, Joyce S, Anil S. Supportive periodontal therapy - is the patient compliance adequate? J Int Oral Health. 2015; 7(11): 16-19.

11. Lopes R, Frossard W, Moreira Â, Fraga R. Reabilitação oral em pacientes periodontais: seriam os implantes a meihor solução? Revista Dental Press De Periodontia E Implantologia. 2010; 4(4): 57-67. 
12. Tabosa e Silva TP, Miranda CCL, Bucco Jr RLS. Rehabilitation of atrophic maxilla without a reconstructive approach. A case report. Dental Press Implantol. 2014;8(2):70-7.

13. Mericske-Stern R, Worni A. Optimal number of oral implants for fixed reconstructions: A review of the literature. Eur J Oral Implantol. 2014. 2: 133-153.

14. Laurito J. Passividade da prótese sobre implante. Innov Implant J. 2010; 5(3): 53-59.

15. Drago C, Howell K. Concepts for designing and fabricating metal implant frameworks for hybrid implant prostheses. J Prosthodont 2012; 21(5): 413424.

16. TaeHyun K, Bain P, Levin L. Systematic review of short- (5-10 years) and long-term (10 years or more) survival and success of full-arch fixed dental hybrid prostheses and supporting implants. J Dent. 2014; 42(10): 1228-1241.
17. Yamazaki K. A retrospective comparative ten-year study of cumulative survival rates of remaining teeth in large edentulism treated with implant-supported fixed partial dentures or removable partial dentures, J Prosthodont Res. 2013;57: 156-161.

18. Sailer I, Muhlemann S, Zwahlen M, Hammerle CHF, Schneider D. Cemented and screw-retained implant reconstructions: a systematic review of the survival and complication rates. Clin Oral Impl Res. 2012; 23:163-201.

19. Linkevicius T, Puisys A, Vindasiute E, Linkeviciene L, Apse P. Does residual cement around implantsupported restorations cause peri-implant disease?: A retrospective case analysis. Clin Oral Impl Res. 2012; 1: $1-6$.

20. Kim Y, Oh T, Misch CE, Wang H. Oclusal consideration in implant therapy: clinical guidelines with biomechanical rationale. Clin Oral Impl Res. 2005; 16:26-35.

Recibido: 14-06-19

Aceptado: 25-03-20 